\title{
RCSB Protein Data Bank: Celebrating 50 years of the PDB with new tools for understanding and visualizing biological macromolecules in 3D
}

\author{
Stephen K. Burley \\ Rutgers, The State University of New Jersey, Piscataway, United States of America; \\ sburley@proteomics.rutgers.edu
}

The Research Collaboratory for Structural Bioinformatics Protein Data Bank (RCSB PDB), the US data center for the global PDB archive and a founding member of the Worldwide Protein Data Bank partnership, serves tens of thousands of data depositors in the Americas and Oceania and makes 3D macromolecular structure data available at no charge and without restrictions to millions of RCSB.org users around the world, including $>800000$ educators, students and members of the curious public using PDB101.RCSB.org. PDB data depositors include structural biologists using macromolecular crystallography, nuclear magnetic resonance spectroscopy, 3D electron microscopy and micro-electron diffraction. PDB data consumers accessing our web portals include researchers, educators, and students studying fundamental biology, biomedicine, biotechnology, bioengineering, and en ergy sciences. During the past two years, the research-focused RCSB PDB web portal (RCSB.org) has undergone a complete redesign, enabling improved searching with full Boolean operator logic and more facile access to PDB data integrated with $>40$ external biodata resources. New features and resources will be described in detail using examples that showcase recently released structures of SARS-CoV-2 proteins and host cell proteins relevant to understanding and addressing the COVID-19 global pandemic.

Keywords: Protein Data Bank, Macromolecular Structure, Open Access 